\title{
La idea de universidad en vilo. Gestión de calidad, capitalismo cognitivo y autonomía
}

The Idea of the University on Tenterhooks. Quality Management, Cognitive Capitalism, and Autonomy

A ideia de universalidade em suspense. Gestão de qualidade, capitalismo cognitivo e autonomia

\section{Esther Juliana Vargas Arbeláez ${ }^{*}$}

\section{Artículo de investigación}

Revista Colombiana de Educación, N.7 72. Primer semestre de 2017, Bogotá, Colombia.

Para citar: Vargas, E. J. (2017). La idea de universidad en vilo. Gestión de calidad, capitalismo cognitivo y autonomía. Revista Colombiana de Educación, (72), 139-157.

Fecha de recepción: 02/08/2016

Fecha de acpectación: 29/09/2016

* Docente y gestora de proyectos de la Universidad del Rosario. Licenciada en Filosofía de la Universidad de San Buenaventura; MA. in Book and Digital Media Studies de Leiden University; Estudiante de Doctorado en Filosofía de la Universidad de Antioquia- Colombia. Correo: estherjulianava@yahoo.com.ar 


\section{Resumen}

El propósito de este texto es examinar la manera en que las prácticas de gestión de calidad amenazan la autonomía universitaria y -asumiendo esta como el rasgo definitorio de la universidad- se discurre sobre la puesta en vilo de la idea de universidad misma en tiempos de capitalismo cognitivo. Para este efecto, el texto tiene tres partes: en primer lugar se repasa la forma en que la gestión de calidad se inserta en la pretensión de excelencia de la universidad como discurso teleológico que sostiene las prácticas productivas de ciencia, ordenadas a satisfacer las necesidades del mercado. En segundo lugar, se repasa la tesis del capitalismo cognitivo y su efecto en las políticas de ciencia, marcadas por los dispositivos como el de la gestión de calidad. Por último, se plantea la cuestión sobre la autonomía como idea reguladora y constitutiva de la universidad y la renovación de la lucha por su conquista en tiempos de capitalismo cognitivo.

\section{Palabras clave:}

capitalismo cognitivo, autonomía universitaria, gestión de calidad.

\section{Abstract}

This paper aims to examine how the practices of quality management have threatened university autonomy and also-assuming that it is the defining feature of the university-how it has put on tenterhooks the very idea of the university in times of cognitive capitalism. For this purpose, the paper is divided into three parts. The first part examines the way quality management was inserted into the university's ambition of excellence as a teleological discourse, which consequently Reeps production practices of science established under market needs. The second part reviews the theory of cognitive capitalism and its effects on science policies, marked by devices such as quality management. Finally, the last part considers the problem of autonomy as a regulatory and constitutive idea of the university and the renewal of the fight to conquer it in times of cognitive capitalism.

\section{Resumo}

O proposito deste texto é examinar a forma em que as práticas de gestão de qualidade ameaçam a autonomia universitária e -assumindo-a como o rasgo que define a universidade- se reflete sobre o risco em que está a ideia de universidade na época do capitalismo cognitivo. Para esse propósito, o texto divide-se em três partes: primeiro, repassa-se a forma em que a gestão de qualidade é inserida na pretensão de excelência da universidade como discurso teleológico que afirma que as práticas produtivas de ciência ordenadas para satisfazer as necessidades do mercado. Segundo, analisa-se a tese do capitalismo cognitivo e seu efeito nas políticas de ciência, marcadas pelos dispositivos como o de gestão de qualidade. Finalmente, questiona-se a autonomia como ideia reguladora e constituinte da universidade e a renovação da luta pela sua conquista em época do capitalismo cognitivo.

\section{Palavras chave}

Capitalismo cognitivo, autonomia universitária, gestão de qualidade 
No se tratará tanto de una tesis, en verdad, ni siquiera de una hipótesis, cuanto de un compromiso declarativo, de una llamada en forma de profesión de fe: fe en la universidad y, dentro de ella, fe en las Humanidades del mañana.

J. Derrida (2002).

\section{Introducción}

¿De qué manera se configuran los dispositivos (Agamben, 2011) de medición de calidad (estándares de evaluación, bases de datos e índices de revistas, mediciones de audiencia, reconocimiento de investigadores y grupos de investigación, y políticas de ciencia estatales)? ¿A qué le apuestan estos dispositivos? ¿Cuál es la idea de universidad que encarnan y cuál idea de ciencia está en la base de estas apuestas? ¿Qué queda adentro de la ciencia? ¿Qué saberes, temas, procedimientos y dinámicas científicas se excluyen a partir de las políticas de ciencia?

En los últimos años hemos visto en las universidades la inserción inexorable de mecanismos de la gestión de calidad que fueron creados y, estructuralmente, son propios de los ambientes fabriles y de las empresas. Como consecuencia, actualmente hay quienes hablan del fenómeno de la universidad-fábrica (Restrepo, 2012, 2013; Edufactory-Uninomade, 2010; entre otros), que no solo intenta estar en correspondencia con los requerimientos del mercado, sino que persigue la validación estatal, con políticas de ciencia -a la postre- ordenadas a esos requerimientos del mercado, o de los rankings internacionales bajo las banderas de la excelencia y el mérito.

La tesis que se quiere defender en este texto es que la gestión que calidad-que promueve la excelencia (como la critican, por ejemplo Vessuri, Guédon y Cetto, 2013) o se basa en meritocracia (en el análisis de Roggero, 2011)- que orienta las políticas de ciencia estatales, están basadas en criterios por fuera de las prácticas científicas y encarnan la tesis del capitalismo cognitivo. Más aún, este sistema pone en vilo la autonomía universitaria -suerte de leitmotiv de esta institución del saber-y renueva la necesidad de una lucha por la defensa de los linderos de la universidad. En suma, en este texto se quiere exponer la necesidad de una renovación de la idea de universidad como forma de antítesis de los criterios de eficiencia y satisfacción del cliente que cercan sus prácticas, sus aspiraciones y su definición. 


\section{La gestión de calidad y la universidad-fábrica}

El sistema de gestión de la calidad es una de las grandes conquistas teóricas y prácticas del campo disciplinar de la administración de empresas. Su éxito como estructura explicativa y prefigurativa de las tareas productivas es tal que, sin crítica conocida o al menos no suficientemente vehemente, se ha implementado de forma total en casi todos los campos de las actividades productivas, así como en otras dimensiones humanas que no tienen, por su naturaleza, una relación directa con el capital; pero están conminadas a sistematizar y exponer su calidad, tal como el ambiente académico-universitario. Los parámetros de la administración de empresas se cuelan en la academia por vía de la política pública de ciencia y de allí se encarnan en las dimensiones centrales de la universidad, a saber: formación, investigación y extensión.

En el texto La gestión de la calidad en la educación, López Rupérez (1994) explica la centralidad de la gestión de la calidad en la vida contemporánea.

Los países más desarrollados están experimentando un proceso de auge imparable de la valoración social de la calidad. Como si de una palabra mágica se tratara, el término calidad evoca en la mente de las personas la referencia a un valor seguro, se trata de un atributo [...] de los objetos que, según la percepción de los ciudadanos, satisfacen sus expectativas razonables haciéndolos dignos de confianza. (p. 11).

Las razones por las que la idea de calidad ha cobrado tanto valor en la sociedad contemporánea, la explica este autor a partir de tres aspectos: en primer lugar, la calidad "constituye un elemento de marketing de primera magnitud"; por ello, "las empresas están orientando su actividad y los modos de gestionarla hacia la calidad"; lo cual, a la postre, fomenta el "incremento de la competitividad" en el que el 'sello de calidad' es el valor añadido (cf. López, 1994, p. 11).

Estos referentes sociales sobre la calidad no son solo una especie de imaginario colectivo, sino que están regulados por formas estandarizadas que funcionan como regla y como aspiración de cualquier organización productiva ${ }^{1}$. Me referiré, en particular, a la norma ISO9001. Esta tiene el objeto de producir en las empresas una forma convencional de eficiencia basada en los siguientes criterios: establecimiento de estrategias (orientadas a la satisfacción del cliente y que hablan de los lineamientos generales de la empresa), los procesos (alineados con las estrategias), los recursos, la estructura organizacional y, finalmente, tener los documentos de soporte de todo esto.

1 Cuando se trata, no de productos, sino de servicios - de orden cultural o académicoesta idea parece haber sistematizado e instrumentalizado lo que ya Bourdieu (1997, 1998, 2008, entre otros textos) analizaba el siglo pasado sobre el capital cultural y simbólico. Con este dispositivo ya hay cómo medirlo y certificarlo. 
Llama la atención que la norma internacional ISO9001, que además suscriben no solo empresas que ofrecen productos sino también instituciones que ofrecen servicios, inicia el documento regulador con la aclaración de que la norma "promueve la adopción de un enfoque basado en procesos [...] para aumentar la satisfacción del cliente mediante el cumplimiento de sus requisitos [...]; y entiende el proceso como que "los elementos de entrada se transformen en resultados" (Icontec, 2008, p. 9; cursiva por fuera del texto original).

\section{NORMA TÉCNICA COLOMBIANA NTC-ISO 9001 (Tercera actualización)}
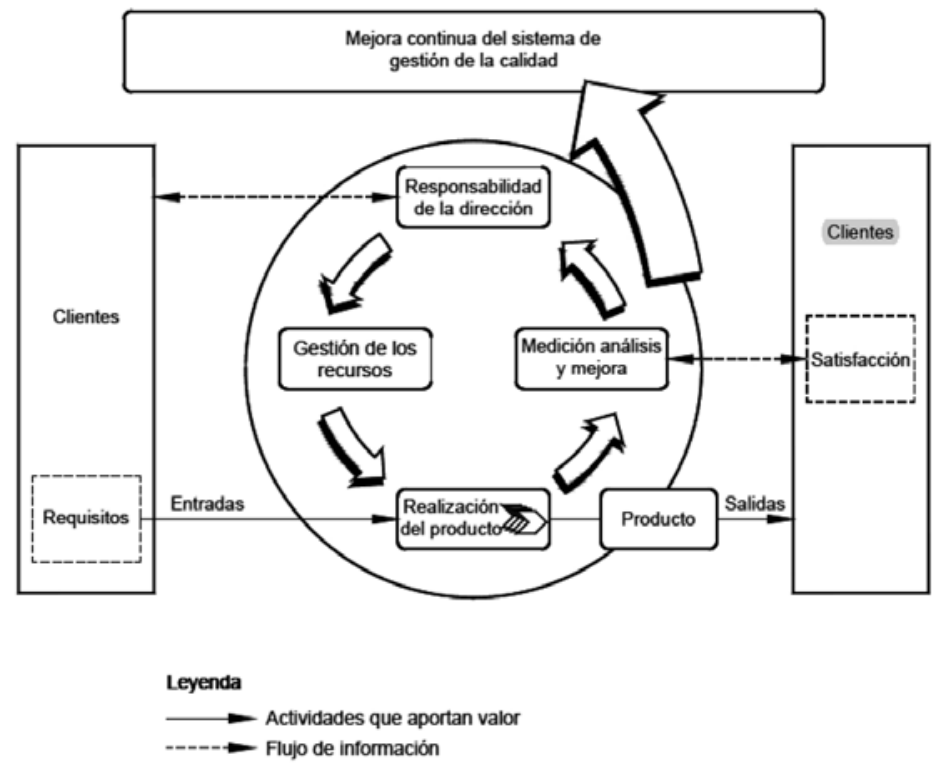

Figura 1. Modelo de un sistema de gestión de la calidad basado en procesos

Fuente: Icontec (2008, p. 10, resaltado por fuera del original)

Quisiera insistir en el hecho de que el sistema de gestión de calidad tiene como fundamento la satisfacción del cliente; y para eso implementa el enfoque basado en procesos que estandariza, anticipa, regula y documenta todos los aspectos de la actividad a la que se le aplica ese sistema de calidad.

Pues bien, la norma ISO9001 es la que regula la calidad de las instituciones de educación superior en Colombia, a pesar de que claramente está concebida como un sistema de monitoreo de la producción de empresas que generan capital (de ahí la centralidad del marketing, del valor agregado $y$, sobre todo, del cliente), y en cambio la universidad, en principio, tiene una naturaleza y pretensiones diferentes a las del lucro, al menos idealmente como institución en la composición de la sociedad. 
¿Acaso la universidad se convirtió en un lugar de generación de capital? Los hechos nos muestran una respuesta afirmativa; ya está ampliamente documentado el fenómeno de la universidad-empresa y de la universidad-fábrica, así como "mercantilización y precarización del conocimiento" (Ferreiro, citado por Edufactory, 2010).

La universidad-empresa abarca el fenómeno de la simbiosis productiva entre la universidad y la cooptación o compra de conocimiento por parte de las empresas privadas -lo cual va en contra de la aspiración del conocimiento como bien común y lo convierte en producto de cambio y lucro (como se examinó en Vargas, 2014)-; promovida, además y desconcertantemente, por los Estados a través de sus políticas públicas.

La universidad-fábrica parece bien descrita, por ejemplo, por Montenegro y Pujol (2013), quienes muestran -en especial pp. 143-146- cómo las prácticas y aspiraciones de la universidad adoptan la forma de ser de la producción en serie de la fábrica. El discurso de la calidad es central en este proceso, atravesado por una de las consecuencias del capitalismo cognitivo que Virno (2003a) ha señalado: el desdibujamiento de los bordes entre el trabajo y la vida privada. En ese sentido:

[...] el hecho de que la subjetividad académica es transformada y redirigida sobre la base de las exigencias del trabajo cognitivo y bajo una ideología centrada en los procesos de calidad, permite comprender la relativa aceptación de las reformas en la organización universitaria y la dificultad de consolidar una resistencia generalizada a su implantación. Nos encontramos en una nueva época donde la producción de conocimiento y la gestión de la afectividad requieren de una deslocalización y fragmentación laboral cuya exigencia borra las fronteras entre trabajo y ocio en aras de un "bien social" general. Efectivamente, en la nueva sociedad del conocimiento la institución universitaria es un productor potencial de significados, procedimientos tecnológicos y de obra de mano cualificada que, además, certifica su conocimiento como "verdadero" e "imparcial". (Montenegro y Pujol, 2013, p. 146).

El carácter de verdadero e imparcial constituye una parte muy importante del discurso de la calidad, en una amalgama que se hace entre la pretensión de cientificidad de los saberes que se tratan en la universidad -asepsia epistemológica- y una despolitización de las prácticas del conocimiento, conveniente a los efectos que persiguen las empresas con ánimo de lucro.

Pero, ¿de qué manera se introduce este discurso en el ámbito educativo en las instituciones del saber? ¿Cuáles son los efectos de la introducción de un sistema fabril en los lugares de la ciencia? ¿Qué implicaciones tiene esto en la idea de ciencia que manejamos en la actualidad? Volveremos sobre esto más adelante. Por ahora vamos a detenernos en la primera pregunta: ¿De qué manera se introduce este discurso en el ámbito educativo de saber científico? 
Una respuesta desde la filosofía de la ciencia se encuentra en las reflexiones que van desde el desconcierto de Heidegger con la técnica moderna (que devela; pero también oculta el ser-Heidegger 1976, 1997, 2002-) hasta la filosofía de la tecnología, atravesada por lo digital, de Broncano (2005); esto sin contar con el ingente esfuerzo por revindicar el estatuto científico de las ciencias sociales y humanas que se tomó gran parte del siglo pasado. La crítica a la racionalidad moderna (instrumental, etc.) ha mostrado de qué manera se configuró el paradigma positivista en la ciencia y de qué forma se prefiguró la comprensión del saber y de otros aspectos humanos basados en las aspiraciones modernas por el cálculo, la descripción, la previsión y la universalización. Con ello, en consecuencia, se moldeó epistemológicamente el conocimiento valorado en los siglos XVIII al XX, a partir de criterios enunciados desde la ciencia que dejaron por fuera los métodos cualitativos y las preguntas de orden de las humanidades y las ciencias sociales. En suma, se asumió una forma de racionalidad como la única forma canónica del conocimiento, con una alta dosis de estandarización como horizonte de validación.

A pesar de los esfuerzos por los replanteamientos epistemológicos que se implementaron a lo largo del siglo pasado, parece que enfrentamos una batalla que se está perdiendo. La tarea de darle a las humanidades y los saberes no-cuantificables un lugar digno en la ciencia, esto es, la tarea de hacer de la ciencia un lugar del pensamiento y no sólo de la producción, parece cada vez más difícil; ya no solo por cuenta de una concepción de ciencia que se enuncia desde dentro de sí misma -con los prejuicios epistemológicos que fundaron la idea de que la ciencia era solo lo probable o lo predecible-; sino a partir de criterios objetivos de medición que están por fuera de la ciencia y que encuentran su fundamento en la administración de empresas y en las leyes del mercado. Pasamos de una aspiración epistemológica de estandarización, a prácticas efectivas "ordenadas a procesos, descriptibles y a satisfacción-medible-del cliente", como se recapituló atrás con la norma de calidad.

Esto es: la ciencia se está prefigurando con una teleología positivista pero renovada, que apunta a la producción de tecnología, conocimientos, códigos, información, u otro tipo de materialización capitalizable, ya no solo por cuenta de la racionalidad moderna -que seguramente está a la base de este fenómeno- sino sobre todo por cuenta de los dispositivos fabriles-empresariales que se han incorporado en su vida diaria. En suma, presenciamos la consolidación de la universidad-empresa y la corporatización de sus actividades relativas al conocimiento científico (tal como ya lo diagnosticaron Edufactory, 2010, 2011; Roggero, 2011; Cuninghame, 2008; entre otros).

Esto sucede de dos formas diferentes: de un lado se puede identificar la incorporación de la productividad en las prácticas de la academia, 
que se materializan en hacer que los profesores sean más eficientes, que produzcan más artículos, que atiendan más estudiantes, que documenten todo esto y lleven registros de cómo administran su tiempo y su trabajo; todo ordenado al sistema de gestión de calidad que impele a que estos procesos sean visibles y se estandaricen.

Basta ver los requisitos para la acreditación de instituciones de educación superior que determina el Ministerio de Educación Nacional de Colombia (que evalúa sus programas, sus procederes, su estructura como empresa). Si bien es cierto que están inspirados en una pretensión de excelencia que no solo se apoya en la evaluación de los procesos, sino que "son más exigentes, pues incorporan fuertemente los procesos misionales, es decir los educativos, los que son incluidos extensamente en la autoevaluación y revisados por pares académicos, tanto para el rector, como para las áreas de conocimiento impartidas, bienestar e infraestructura" (MEN, 2016); también es cierto que la estructura de evaluación está completamente montada sobre los modelos de gestión de calidad -como el de la norma revisada atrás, de orden fabril y empresarial-.

De otro lado, la inserción del proceder fabril se evidencia en los efectos de estas prácticas, o al menos los que se espera de ellas en este modelo; esto es, que los resultados de la investigación puedan producir réditos monetarios reales y contantes a través de patentes o tecnología aprovechable, especialmente, por el sector privado.

Los primeros visos de esto se hallan, de nuevo, con López Rupérez (1994), en la preocupación de finales del siglo pasado por hacer de la educación un vehículo de generación de nueva tecnología a través de la producción masiva de trabajadores competentes para afrontar "los retos del mundo tecnológico".

En la década de los 60 el desarrollo de la carrera del espacio no solo llevó consigo un despegue singular en el ámbito científico-tecnológico, sino que el reto de colocar un hombre en la Luna alcanzó de lleno al mundo de la educación. Se renovaron las expectativas, particularmente, respecto del poder de una educación científica que estuviera a la altura de las exigencias marcadas por los nuevos desafíos, y aparecieron en los países más desarrollados proyectos de reforma curricular orientados a contribuir, desde la educación de base, al progreso científico y tecnológico. (López, 1994, p. 17).

Y aunque en los años 1960 este entusiasmo decayó;

Sin embargo, la década de los 80 se caracterizó por un resurgimiento del interés por la contribución de la educación y de la formación a los resultados de las economías nacionales, y en los actuales 90 educación y formación se han situado entre las primeras prioridades de 
las políticas sociales y económicas de los países de la OCDE. (López, 1994, p. 18).

El autor hace coincidir este resurgimiento con la consolidación de la sociedad del conocimiento y de la información, su relación con el desarrollo económico y la posición estratégica de la educación en este contexto.

Dicho posicionamiento estratégico lo identifican y reconocen bien los Estados. Examinemos el caso colombiano. La política de ciencia y tecnología que está vigente en Colombia está orientada al desarrollo de patentes, de tecnología y de aprovechamiento de los recursos naturales de la nación. En esa política nacional de ciencia, el lugar de las humanidades no es tímido, sino prácticamente inexistente y la idea de ciencia y sus dispositivos (la regulación de las instituciones de educación superior, las publicaciones científicas, la contabilidad de doctores graduados, etc.) está marcada por la claridad en los procesos y un trasfondo de satisfacción del cliente (como lo anhelan las normas de calidad); toda vez que incorpora al sector privado y comercial en las tareas de generación de ciencia y tecnología. Veamos cómo:

Lo primero que se puede considerar es que, en el comienzo mismo del documento Conpes (Consejo Nacional de Política Económica y Social) de la Política de ciencia y tecnología colombiana (2015-2025), el fundamento de la formulación de la política está en el vínculo que se establece entre el desarrollo económico y el desarrollo de la ciencia. Eso implica, entre otras cosas, que los programas y la financiación están orientados a los proyectos de investigación con los cuales la nación pueda ver retorno eficiente y claro en términos de producción de capital.

En la justificación de la política se aborda la relación entre innovación y productividad, con base en evidencia empírica a nivel internacional, encontrando un fuerte vínculo y confirmando la relevancia de la CTI [ciencia, tecnología e innovación] como fuente de crecimiento, que genera beneficios económicos y sociales. (Conpes, 2015, p. 3).

Esto, tal como está concebido en la política nacional de ciencia, debe darse a través de la vinculación de la ciencia con el mercado, no solo aportando este capital, sino también, y sobre todo, beneficiándose de su inversión, naturalmente.

Estas interacciones se dan por mecanismos de mercado así como por fuera de él, por lo que la política de CTI se aborda en respuesta a la resolución de fallas del mercado, así como a problemas sistémicos relacionados, por ejemplo, con fallas institucionales. (Conpes, 2015, p. 15). 
El sector empresarial tiene un lugar protagónico en este documento que consagra la política de ciencia de la nación. Las normativas y procedimientos efectivos en las entidades responsables de producir ciencia deben "hacer efectiva la inversión y lograr apalancar recursos del sector empresarial" (Conpes, 2015, p. 15).

En este sentido, y tal como se afirma en el mismo documento, la política de ciencia colombiana se propone fortalecer la competitividad; para lo cual se insta a optimizar el gasto público, dando la bienvenida a la inversión privada proveniente del sector empresarial. En suma y sin mayores esfuerzos, está clara la orientación de gestión de calidad para satisfacer al cliente (esto es, el sector empresarial). En otros términos, los sistemas de gestión de calidad, del tipo de la norma ISO9001, no solo se convierten en referentes convencionales para las empresas (con la claridad de que la universidad es, de facto y bajo estas aspiraciones, una empresa), sino además en el modelo de regulación de la ciencia que anhela una nación.

No extraña, entonces, el vértigo de las instituciones de educación superior por comprobar que son de calidad y, en consecuencia, implementan ese sistema de gestión. Esto no solo es política de ciencia de la nación colombiana (que encarna lo que el país político-institucional entiende por ciencia, sus apuestas sociales y epistemológicas -en este caso, inexistentes-), sino que define qué universidad entra o sale del elenco de las instituciones acreditadas por el Estado (es decir, condiciona las bases efectivas de la infraestructura de generación de ciencia). ¿Cómo afecta esta preocupación por el sello de calidad Icontec -que es la entidad colombiana que garantiza la calidad, con base en los criterios ISO9001- la naturaleza de la universidad, su dimensión misional?

Otro tanto sucede con los procesos formativos: los estudiantes deben aprender a ser productivos; deben graduarse vertiginosamente y deben ser satisfechos como clientes del sistema formativo. No hay tiempo para una formación humanista. En el documento Conpes se establece una relación entre el capital humano y el incremento de la productividad; entre la profesionalización y la innovación; entre la formación y el aumento del capital líquido de la nación. No se menciona la dimensión humanista de la universidad, su talante como universitas (Vargas, 2013).

También en el horizonte investigativo hay afectaciones: presenciamos y experimentamos la centralidad de la producción en serie de artículos científicos y proyectos de investigación que sirvan para algo. En la escala de valoración de la producción de conocimiento están en primera línea los artículos de investigación con resultados acabados. Muy abajo están los ensayos, artículos de reflexión, discusiones teóricas, etc., tan caros para la comprensión de los horizontes disciplinares. En vez de eso, en el documento Conpes se invita a que la investigación "expanda la 
frontera del conocimiento para dar respuesta al desarrollo productivo del país" (Conpes, 2015, p. 16).

Finalmente, la extensión universitaria: lo que otrora fue la oficina que coordinaba la relación de la universidad con la comunidad, a través de proyectos con impacto social directo, actualmente está concentrada en la generación de estrategias de productividad con la empresa privada para el aprovechamiento y capitalización del conocimiento que se produce en la universidad; lo cual va en correspondencia con el Ilamado nacional a hacer la de la universidad un agente innovador para solventar el problema "del crecimiento de las empresas debido a la baja actividad innovadora" (Conpes, 2015, p. 37).

En este punto quisiera retomar las preguntas que se plantearon atrás:

¿Acaso la universidad se convirtió en un lugar de generación de capital? ¿Cuáles son los efectos de la introducción de un sistema fabril en los lugares de la ciencia? ¿Cuáles son los dispositivos por medio de los cuales se materializa esta aspiración productiva para la ciencia?

\section{Del capital cultural al capitalismo cognitivo. Los dis- positivos}

Ciertamente, la aspiración de la calidad, vista con ingenuidad, no es necesariamente una cosa negativa. ¿Quién podría defender la idea de que la calidad no sea un objetivo de las actividades de la universidad o de la academia? Más aún, este sistema de gestión de calidad parece sistematizar la explicación de Bourdieu (1997, 1998, 2008) sobre el capital cultural de un agente social; esto es, el que puede mostrar confiabilidad para la realización de una tarea académica, cultural, simbólica. ¿En qué se fundamenta contemporáneamente, sin embargo, dicha confianza? ¿Acaso en la producción de conocimiento científico que responde a un programa de investigación de largo aliento; a un problema que se estudiaba por décadas; a la conformación de un equipo-grupo de interesados en un asunto para investigar?

Uno de los elementos que caracterizarían a un investigador como agente reconocido para llevar a cabo su tarea científica, en el marco de la institución universitaria, sería su formación disciplinar así como su formación humanista; pero esta última no está contemplada en la aspiración universitaria actual (en la que vemos en la política pública y de facto, aunque nominalmente siga definiéndose con los cánones modernos); puesto que ella -la formación humanista- implicaría efectos no deseados como el desarrollo de una subjetividad política, el pensamiento crítico, la comprensión holística de las tareas operativas, etc. Claramente esto no genera capital; al contrario, le amenaza. 
Este fenómeno parece materializar la reflexión teórica de Virno sobre la despolitización de la vida pública por vía de la adopción de la estructura de la vida privada en la vida productiva. En Virtuosismo y revolución (2003b), este autor se auxilia del análisis del ejercicio virtuoso del artista que no produce obra cuando se trata del performance, que se consume en el acto artístico: la interpretación en vivo del músico, la presentación del actor en tablas, etc.; y ve en este concepto una estructura explicativa de la acción política: ella se agota en sí misma, en ella, la subjetividad se entrega toda y se consume en su actuar. La acción política tiene la estructura del virtuosismo; en ambos casos (artista y sujeto político) el actuar no "produce" "existencias" o superávit (en el sentido que explica Heidegger (1997) en La pregunta por la técnica, para comprender la exacerbación del emplazamiento del ser que genera la técnica moderna).

Sin embargo, en el análisis de Virno, los tiempos posfodistas nos han mostrado que no solo la acción política debe tener la estructura virtuosa: también la actividad productiva. Dado que la producción de capital depende de estructuras lingüísticas y de productos inmateriales, la subjetividad debe comportarse como el artista virtuoso o como el desencadenador de la acción política: debe actuar sin dejar obra -digamos: no se requiere producto, puesto que no se trata de un obrero fabricante de objetos-, debe agotarse en el hacer -digamos: en la generación de contenidos informacionales o de conocimiento-. Sin embargo, a diferencia del virtuoso (artista o sujeto político), el trabajador-productor-de-capital está actuando prosaica y precariamente. No se pregunta, no se cuestiona: está envuelto en el hacer productivo que invade su vida privada en tiempo y en estructura (cf. Virno, 2003a).

Estos elementos forman parte del aparato teórico de la tesis del capitalismo cognitivo: se trata de una economía basada en el conocimiento -se transan bienes inmateriales derivados de la producción de saberque, para ser efectiva, tiene control biopolítico sobre los sujetos -quienes son productivos incluso en los ratos de ocio, conscientemente o no- y requiere que se consolide la despolitización de la actividad humana -se debilita la subjetividad política y se fortalece la idea de que los mecanismos institucionales de ciudadanía a primera mano, como el voto, agotan lo que un sujeto debe hacer sobre esta materia-.

Vercellone (2007) explica este asunto así: "La hipótesis del capitalismo cognitivo deriva de la crítica a la economía política de las nuevas teorías liberales de la economía basada en el conocimiento" (p. 14, traducción libre). La estructura capitalista actual depende de la producción de conocimiento, información o códigos que circulan y se consumen -ideas, tecnología, símbolos, etc.-. El trabajo inmaterial se define como "la labor que produce el contenido informacional y cultural de una mercancía" (Lazzarato, 1996, p. 133) que pasa a tener un lugar menos relevante en 
la producción efectiva de capital, en comparación con lo que produce el conocimiento mismo. Por eso es que Vercellone habla de una economía basada en el conocimiento para explicar el fenómeno posfordista.

¿Acaso esta teoría traída del pensamiento filosófico (de la filosofía política y de la economía política) tiene, en efecto, un correlato en la actividad real productiva en la universidad? Ciertamente. Como se revisó atrás, la relación estrecha entre producción de capital y producción de saber sí se ha instrumentalizado a partir de políticas y de prácticas concretas.

En un artículo de 1997, Nelson y Romer $^{2}$ analizan la centralidad del wetware y evocan la necesidad de una intervención estatal, no tanto para financiar la investigación y el desarrollo de las empresas sino directamente el wetware, el capital humano formado en las universidades. Los tiempos de formación, los tiempos de comunicación, los tiempos de construcción de redes semánticas y sociales ahora parecen ser reconocidos implícitamente como tiempos de producción de riqueza esenciales en la producción contemporánea de valor. (Corsani, en AA.VV, 2004, p. 95).

Todo esto es posible, si queremos el auxilio de la teoría, gracias a la eficiencia de los dispositivos (Agamben, 2011) ( $^{3}$ que, en este caso, cumplen con la misión de aterrizar en las prácticas concretas de los productores de conocimiento científico: tanto los megadiscursos sobre la relación entre la investigación en la universidad, la formación del capital humano y el desarrollo; así como la homogénea pretensión de excelencia que sostiene dicha relación.

Esto se puede ilustrar con una de las actividades científicas centrales de la universidad y que más réditos meritocráticos les trae a estas instituciones: la producción académica publicada (tanto las revistas universitarias, en general, como los artículos de los profesores, en particular). Las publicaciones científicas funcionan como dispositivo bisagra entre la política de ciencia y las prácticas reales de los investigadores. La altísima estandarización de la valoración calidad en las publicaciones científicas a partir de los indicadores basados en la cita funciona como parámetro para medir por igual a personas, grupos de investigación, instituciones y programas que estas ofrecen; además de generar un referente para decidir qué se financia y qué no.

2 Se refiere al texto de Nelson y Romer (1996).

3 Agamben describe el dispositivo como "un conjunto de prácticas y mecanismos (invariablemente, discursivos y no discursivos, jurídicos, técnicos y militares) que tiene por objetivo enfrentar una urgencia para obtener un efecto más o menos inmediato" (Agamben, 2011, p. 254). 
Lo curioso es que esta estrategia de medición-valoración, como lo explican Verssuri et al. (2013), no nació como un sistema de estratificación de la calidad de la ciencia, sino como una forma de mapeo, de compresión de la actividad científica como -digamos- actividad social. En este sentido explican:

Originalmente, la aplicación de los indicadores basados en la cita fue diseñada para delinear la circulación de teorías, conceptos, métodos y herramientas, para analizar las conexiones entre los científicos y mesurar el impacto de los artículos publicados. Diseñados para entender la red de comunicaciones de la ciencia, y para ayudar la heurística interdisciplinaria, estos indicadores no estaban destinados a medir la calidad. (Vessuri, Guédon y Cetto, 2013, p. 2; traducción libre) $)^{4}$

A pesar de ello, y gracias a su estructura formal adecuada, estos indicadores migraron su inspiración original, a la de servir de instrumentos para juzgar la ciencia y, con ello, encarnan la función de bisagra como dispositivos de la política estatal sobre las prácticas de ciencia. Más aún, no solo operan como un sistema de evaluación -con el vértigo imperante que ejerce esto sobre todos los actores que tienen prácticas y preocupaciones al margen de estos sistemas de evaluación-, sino que están ordenados al dictamen del mercado: bien es sabido que la normalización de indicadores basados en citas, de los índices y de los correspondientes cuartiles, ha sido una práctica propia de las editoriales comerciales que fueron las primeras en estandarizar estos mecanismos de gestión y valoración de calidad en las publicaciones (Guédon, 2001, pp. 145 y ss.). También ha sido criticada la relación entre esta normalización y la renta subsecuente.

Esto no solo pone en cuestión lo que estamos entendiendo por ciencia, sino que además ofrecería un manto de duda sobre las motivaciones que inspiran el contenido del título calidad en este contexto -o del título excelencia, como analizan Vessuri, Guédon y Cetto (2013)-. ¿Llamamos ciencia a lo que las editoriales comerciales deciden que es tal, esto es, a lo que ellas mismas deciden publicar y valorar? Por otro lado, el dispositivo funciona bien en términos de producir réditos para el mundo empresarial: ¿Llamamos ciencia solo a lo que puede ser aprovechable por el sector privado?

Este ejemplo ilustra la relación entre lo que se hace en la universidad y la actual sumisión de su práctica puesta al servicio del lucro privado. Con él se quiere poner en evidencia el cerco que se cierne sobre la universidad, sobre sus prácticas y sus aspiraciones.

4 Aquí se está citando el preprint que está en acceso abierto, no la versión publicada por la revista que está bajo restricciones de pago por parte del lector. 


\title{
Leitmotiv: autonomía universitaria
}

\begin{abstract}
Primero se las denominó [a las universidades] Studia por la familiaridad semántica de este término con el poder del Studium. Ambos términos, recordemos, proceden del verbo latino studeo o vivir apasionado por algo. En este caso, por el poder del saber individual e institucional.
\end{abstract}

A. Borrero (2005).

El problema de la autonomía universitaria es, como tal, una preocupación que no nace nominal o temáticamente en el medioevo, junto con la universidad; sino un problema pragmático que impele a la universidad primitiva -como la llama Borrero- a estructurar definiciones de sus actividades, su estatuto y sus aspiraciones. Alfonso Borrero (2005) afirma: "[E]n las fuentes primarias de la historiografía universitaria, citadas y leídas en obras de muy autorizados historiadores de la universidad, no he encontrado la expresión autonomía universitaria" (p. 4) y que solo aparece el título auctorista para reconocer el "individual derecho autónomo del saber demostrado por los más connotados maestros" (p. 4).

A pesar de esto, las primeras definiciones de la universidad parecen estar invocando el derecho a su autonomía, desde el principio. En el medioevo se identifica bajo el título universitas ${ }^{5}$ a la corporación o "grupo sociológico determinado" (Borrero, 2005, p. 39) bajo la necesidad, primero, de diferenciar el gremio de estudiantes y profesores dedicados al saber, de otros gremios (Borrero, 2008, pp. 35 y ss.) -la expresión, según el autor, data del año 1229-; y luego por cuenta de las tensiones políticas que cercaron este originario apasionamiento por el saber (del que habla Borrero, apelando a la etimología). Dichas tensiones provinieron por igual del clero como de la corte; ambos estamentos con la pretensión de hacer de la universidad un instrumento de control, respectivamente, de las almas o de los súbditos.

Estos cercamientos de la actividad consuetudinaria dieron el impulso efectivo para las definiciones de universidad; esto es, la idea de universidad parece haber requerido, desde el comienzo, de un agente exógeno que le hiciera objetivar su ser en discursos y definiciones más formales y no solo en prácticas materiales. En otros términos, la universidad requiere de la defensa de su autonomía como leitmotiv de su definición.

5 Sobre el peregrinaje conceptual y nominal que va desde la designación studium hasta universitas (véase Borrero, 2005, pp. 38-41). 
Un ejemplo de esto es la prohibición decretada por el obispo Tempier contra la enseñanza de la filosofía aristotélica en la Universidad de París. Esta prohibición tuvo como efecto la declaración formal de lo que se enseñaba (y de lo que no debía enseñarse, a su juicio) en la universidad; una suerte de declaración de los contenidos de lo que hoy llamaríamos currículo. Cabe resaltar que esto sucedió luego de por lo menos medio siglo de actividad intelectual consuetudinaria en la Universidad de París ${ }^{6}$.

Más adelante, la idea de universidad parece reivindicarse nuevamente por vía de la necesidad de la defensa de su autonomía. Baste recordar las posturas kantianas en la Modernidad sobre esta materia, así como las defensas del primado de la razón sobre la heteronomía epistemológica heredada del medioevo (en versión -anterior a lo de Kant- de Pico della Mirandola, por ejemplo).

En el siglo XIX sucede otro tanto.

Al parecer, constituidos en el siglo XIX los Estados modernos, los Estados-nación, percibieron que para lograrlo en lo político y económico, les era también necesario el poder del saber, y en una u otra forma pusieron mano en la universidad, y se originaron los modos universitarios decimonónicos, distinguidos, en principio, por cuál fuera para cada uno la misión prioritaria: la formación de la persona; el avance de la ciencia; o el servicio a la sociedad o al Estado. (Borrero, 2005, p. 4).

Nuevamente, hizo falta una defensa de la autonomía universitaria para comprender su estatuto y su contenido, para defender su incondicionalidad.

¿Cuáles son, ahora, los cercos de la idea de universidad en la actualidad? ¿A qué tarea se enfrenta la autonomía universitaria como idea reguladora y escudo del apasionamiento por el saber? Como se vio en las dos secciones anteriores: la tarea es la de la redefinición de la universidad frente al cerco del mercado; este es su contrario, su alter que, en el ejercicio de cooptación, impele a la universidad a la defensa de la autonomía y, con ello, a revisar el contenido del título idea de universidad.

La universidad enfrenta la necesidad de defender la verdad-cualquiera sea este su contenido-, de defender su "libertad incondicional de cuestionamiento y de proposición" (Derrida, 2002, p. 10). Pero esto es una idea reguladora.

Esta universidad sin condición no existe, de hecho, como demasiado bien sabemos. Pero en principio y de acuerdo con su vocación declarada, en virtud de su esencia profesada, esta debería seguir siendo un términos de las motivaciones políticas -no solo intelectuales- de esta sentencia. 
último lugar de resistencia crítica -y más que crítica- frente a todos los poderes de apropiación dogmáticos e injustos. (Derrida, 2002, p. 12).

Frente a ello, la universidad puede tener dos alternativas: el éxodo que configura la universidad en los bordes (como lo propone Roggero, 2011, o como lo analiza Restrepo, 2012, o en Castrillón, 2013); o la redefinición/reapropiación en defensa de la autonomía, contra el cerco del mercado, dentro de los linderos de la universidad.

La primera opción tiene materializaciones concretas y operativas; en efecto, existen universidades por fuera de los cánones institucionales que heredamos de la Modernidad (un ejemplo de ello es la Universidad Nómada). Pero, ¿no será tarea de la universidad redefinirse en virtud de la defensa de su autonomía, pero también en defensa de su lugar institucional en la organización de la sociedad? ¿Acaso no se necesita un resquicio crítico pero institucionalizado en el vértigo de la sociedad productiva?

Derrida (2002, p. 13) muestra que esta parece ser una tarea de las humanidades pero no defiende necesariamente su estatuto institucional. ¿Cuáles serían las apuestas en este escenario? Habría, quizá, que construir unas formas contra la excelencia -en este sentido administrativocorporativizado, que atiendan la autonomía del saber y de sus prácticas y que apelen nuevamente al leitmotiv que ha servido de constante definición y de horizonte teleológico a la idea de universidad.

\section{Referencias}

AA.VV. (2004). Capitalismo cognitivo, propiedad intelectual y creación colectiva. Madrid: Editorial Traficantes de Sueños.

Agamben, G. (2011). ¿Qué es un dispositivo? Revista Sociológica, 26(73), 249-264.

Borrero C., A. (2005). La autonomía universitaria. Breve ensayo histórico y teórico. Uni-pluri/versidad, 5(1).

Borrero C., A. (2008). La universidad. Estudio sobre sus orígenes, dinámicas y tendencias. Tomo I. Bogotá: Editorial Pontificia Universidad Javeriana.

Bourdieu, P. (1997). Capital Cultural, Escuela y Espacio Social. México: Siglo XXI Editores.

Bourdieu, P. (1998). La distinción. Criterio y bases sociales del gusto. Trad. de Ma. del Carmen Ruiz de Elvira. Madrid: Taurus.

Bourdieu, P. (2008). Homo academicus. Buenos Aires: Siglo XXI Editores.

Broncano, F. (2005). La agencia técnica. Revista de Ciencia, Tecnología y Sociedad, 2(5), 95-107. 
Castrillón, L.A. (comp.) (2013). La universidad por hacer. Perspectivas posthumanistas para tiempos de crisis. Medellín: Universidad Pontificia Bolivariana.

Cuninghame, P. (2008). Edufactory: precarización de la producción del conocimiento y alternativas. Bajo el Volcán, 7(13), 11-24.

Consejo Nacional de Política Económica y Social (Conpes) (2015). Política nacional de ciencia, tecnología e innovación 2015-2025. Documento borrador. Bogotá.

Derrida, J. (2002). La universidad sin condición. Madrid: Editorial Trotta.

Edufactory-Uninomade (2010). La universidad en conflicto. Capturas y fugas en el mercado global del saber. Madrid: Editorial Traficantes de Sueños.

Edufactory Collective (2011). University Struggles and the System of Measure. Edufactory Journal, 1(3-6). Recuperado de: http://eipcp.ne$\mathrm{t} / \mathrm{n} / 1317065729$

Guédon, J.C. (2011). El acceso abierto y la división entre ciencia "principal" y "periférica". Revista Crítica y Emancipación, (6), 135-180.

Heidegger, M. (1976). Sólo un Dios puede salvarnos. Revista de Occiden-

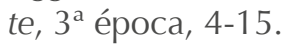

Heidegger, M. (1997). Filosofía, ciencia y técnica. Santiago de Chile: Editorial Universitaria.

Heidegger, M. (2002). ¿Qué es metafísica? Madrid: Alianza Editorial.

Instituto Colombiano de Normas Técnicas y Certificación (Icontec) (2008). Norma técnica Colombiana ISO900. Sistemas de gestión de calidad. Bogotá.

Lazzarato, M. (1996). Immaterial Labor. En: P. Virno y M. Hardt (eds.) (1996). Radical Thougth in Italy. A Potential Politics (pp. 133-150). Minnesota: University of Minnesota Press.

López Rupérez, F. (1994). La gestión de calidad en educación. Madrid: Editorial La Muralla.

Ministerio de Educación Nacional (MEN) (2016). Certificación y acreditación. Recuperado de: http://www.mineducacion.gov.co/1759/w3-article-179263.html

Montenegro M., M. y Pujol T., J. (2013). La fábrica de conocimientos: in/ corporación del capitalismo cognitivo en el contexto universitario. Athenea Digital, 13(1), 139-154.

Nelson, R. y Romer, P. (1996). Science, Economic Growht, and Public Policy. Challenge, 14.17 
Restrepo, C.E. (2012). La universidad en las brumas del capitalismo cognitivo. Recuperado de: http://www.universidad.edu.co/index. php?option=com_content $\&$ view $=$ article $\& i d=2931$ :la- universidad-en-las-brumas-del-capitalismo-cognitivo\&catid=36:ensayos-acadcos\&ltemid $=81$

Roggero, G. (2011). The production of living knowledge. Philadelphia: Temple University Press.

Thijssen, H. (2016). Condemnation of 1277. En: The Standford Encyclopedia of Philosophy. Recuperado de: http://plato.stanford.edu/archives/ fall2016/entries/condemnation/

Vargas A., E.J. (2014). Acceso abierto e instituciones de lo común. Acción política en la academia latinoamericana. Revista Crítica y Emancipación, (12), 357-400.

Vargas G., G. (2013). La Humanitas como Universitas. En: L.A. Castrillón (dir.). La universidad por hacer. Perspectivas poshumanistas para tiempos de crisis (pp. 21-44). Medellín: Editorial Universidad Pontificia Bolivariana, Fondo Editorial Universidad Católica de Oriente.

Vercellone, C. (2005). The hypothesis of cognitive capitalism. Presentado en la Historical Materialism Annual Conference. Birkbeck CoIlege y SOAS, UK. Recuperado de: http://hal.archives-ouvertes.fr/ docs/00/27/36/41/PDF/The_hypothesis_of_Cognitive_Capitalismhall. pdf

Vercellone, C. (2007). From Formal Subsumption to General Intellect: Elements for a Marxist Reading of the Thesis of Cognitive Capitalism. Historical Materialism, 15(1), 13-36. Brill Academic Publishers

Vercellone, C. (2011). Capitalismo cognitivo, renta, saber y valor en la época posfordista. Buenos Aires: Prometeo.

Vessuri, H.; Guédon, J.C. y Cetto, A.M. (2013). Excellence or quality? Impact of the current competition regime on science and scientific publishing in Latin America and its implications for development. Current Sociology. [Preprensa].

Virno, P. (2003a). Gramática de la multitud. Para un análisis de las formas de vida contemporánea. Madrid: Editorial Traficantes de Sueños.

Virno, P. (2003b). Virtuosismo y revolución. La acción política en la era del desencanto. Madrid: Editorial Traficantes de Sueños. 